\title{
Psychosocial characteristics pattern correlated with HIV-related risky sexual behavior among HIV- negative men who have sex with men: a latent profile analysis.
}

\author{
Mengxi Zhai \\ Wuhan University \\ Zhizhou Duan \\ Wuhan University \\ Jiawei Tian \\ Wuhan University \\ Qingqing Jiang \\ Wuhan University \\ Biao Zhu \\ Wuhan University
}

Chenchang Xiao

Wuhan University of Science and Technology

Bin $\mathrm{Yu}$

wuhan university

Hong Yan ( $\nabla$ yanhmjxr@whu.edu.cn )

Wuhan University

\section{Research Article}

Keywords: HIV-negative MSM, HIV-related risky behaviors, Psychosocial characteristics, Latent profile analysis, sex partner, condom use

Posted Date: November 3rd, 2021

DOI: https://doi.org/10.21203/rs.3.rs-983524/v1

License: (c) (1) This work is licensed under a Creative Commons Attribution 4.0 International License. Read Full License 


\section{Abstract \\ Background}

Men who have sex with men (MSM) have become a high-risky population of HIV infection due to their risk sexual behaviors. The psychosocial characteristics play an important effect on HIV-related risky behaviors. This current study aimed to explore the relationship between HIV-related risky behaviors and the latent pattern of psychosocial characteristics among HIV-negative MSM.

\section{Method}

Participants were recruited from Wuhan, Nanchang, and Changsha city from September 2017 to January 2018. The basic socio-demographic characteristics, psychosocial characteristics and HIV-related risky behaviors were collected by self-administered questionnaire and standardize scales. Latent profile analysis (LPA) was used to create the latent classes using variables associated with HIV-related risky behaviors, including social support, reliance, sexual minority stigma, identity concealment, adverse childhood experience and depression.

\section{Results}

Three psychosocial characteristic patterns were revealed by the LPA. "identity concealment group" (IC group) and "ACE group" represented the highest risk profile with highest score of identity concealment and adverse childhood experience (ACE), respectively. "IC group" have a higher likelihood of one-night male partners $(A O R=2.74,95 \% \mathrm{Cl}=1.54,4.90)$, both fixed and one-night male partners ( $A O R=2.01$, 95\% $\mathrm{Cl}=1.34-3.01)$ and $\mathrm{HIV}$-unsure male partner $(\mathrm{AOR}=2.12,95 \% \mathrm{Cl}=1.44-3.13)$ compared with "social support and resilience group" (SR group). ACE group were more likely having inconsistent condom use ( $A O R=2.58,95 \% \mathrm{Cl}=1.41-4.73$ ), and having sex with HIV-positive male partner ( $\mathrm{AOR}=4.90,95 \% \mathrm{Cl}=1.95$ 12.30) with comparison of SR group. Interesting, ACE group had a higher ratio (90.0\%) of inconsistent condom use among MSM whose male partners were HIV-positive.

\section{Conclusions}

Six important psychosocial factors were divided into three latent pattern classes. Compared with "social support and reliance group", "identity concealment group" and "ACE group" were more likely to engage in HIV-related risky sexual behaviors. Besides mental health guidance, promoting serological disclosure of sexual partners and consistent condom use behavior is critical for MSM with higher level of identity concealment or ACE.

\section{Introduction}


Men who have sex with men (MSM) have become a high-risky population of HIV infection both China and the world [1-3]. Previous studies have revealed that the HIV infection has been up to $6.5 \%$ among MSM and it has led to a great burden around the world [4-6]. And, it was reported that the rate of same-sex sexual intercourse has reached $28.2 \%$ in new HIV infections [7]. Similarly, MSM have been focus population in China due to large number of MSM and high rate of new HIV infection. Ying Wang's study in 2017 reported that there were 10-25 million gay men in China which was only a part of all MSM due to their identity concealment [8]. In addition, the proportion of MSM has increased from $61.7 \%$ in 2011 to 73.9\% in 2016 among newly identified HIV cases in China [9]. Katie Huynh 's research showed that from 2010 to 2014, the rate of new HIV infection in the younger MSM population increased by 56\%[10].

HIV-related risky sexual behaviors were a crucial factors of HIV transmission among MSM[11]. A large amount of research indicated that multiple sexual partners, male partner type, inconsistent condom use can increase the risk of HIV infection [12-14]. Furthermore, HIV status of male sex partner and sex after drinking play an important role in HIV risk assessment $[15,16]$.

HIV-related risky behaviors were associated with psychosocial factors including depression, social support, resilience and so on $[17,18]$. In Ahaneku's study, depression was positively correlated with risky sexual behaviors and in turn increased 1.84 times HIV infection risk [19]. In annual cross-sectional survey, Bertolino and his colleges found that MSM having adverse childhood experience (ACE) were more likely to self-report condomless anal intercourse, which was 1.13 times than those participants who didn't experience ACE [20]. Social support and resilience have been confirmed to be protective factor of HIVrelated risky behaviors [21, 22]. In addition, the increasing researches have revealed that sexual minority stigma and identity concealment was positively associated with a number of HIV-related risky behaviors, e.g. it was 1.28 times more likely to have unprotected anal sex when MSM had higher levels of sexual concealing and stigmatization $[23,24]$.

The influence of single psychosocial characteristic on risky sexual behavior had been revealed, but many psychosocial factors coexist and can influence each other. It is unclear how the combined effects of multiple psychosocial characteristics act on HIV-related risky sexual behavior, particularly in HIV-negative MSM. Traditional variable-centered analysis failed to reveal the potential information ignoring the heterogeneity and cumulative effect of psychosocial characteristics. Latent profile analysis (LPA) was a person-centered approach exploring the particular patterns of the population. Therefore, in this study, we firstly used this method to reveal the latent class patterns of psychosocial characteristics; secondly, we further to explore the association of latent pattern of psychosocial characteristics and HIV-related risky sexual behaviors among HIV-negative MSM.

\section{Methods}

\section{Sample and procedure}


With the help of local MSM organization, participants were recruited by peer recommendation and routine HIV test services from September 2017 to January 2018 in Wuhan, Changsha and Nanchang, which all were provincial capital of central provinces in China. More details please see published articles[25-27]. In this study, the inclusion criteria of were: 1) man and were over 16 years old; 2) have sex with man actively in the past six months; 3 ) self-reported that HIV status were negative; 4) agree to provide informed consent. A total of 800 participants were recruited and 749 completed questionnaires, with a response rate of $93.6 \%$. Of all respondents, 160 were excluded for having not sex with man in the past six months and 589 were included in statistical analysis.

\section{Measurements}

\section{socio-demographic variables}

Basic socio-demographic variables including age, ethnicity (Han and others), educational level (High school or lower and College or higher), marital status (Unmarried and Married/divorced), employment status (Unemployed and Employed), monthly income (<1000 Yuan; 1000-3000 Yuan; 3001-6000 Yuan; $>6000$ Yuan) and sexual orientation (Homosexual; Bisexual; Unsure/heterosexual) were collected.

\section{Psychosocial variables}

Sexual minority stigma was assessed by Assessment of Stigma towards Homosexuality (China Version) [28]. It is consisted of three subscales, including perceived stigma, enacted stigma, and the other stigma. The total score ranges from 10 to 40 and higher sum score indicates higher level of sexual minority stigma. The Chinese version of the scale has been confirmed applicable for Chinese MSM population, and Cronbach's alpha was 0.74 in this study.

Adverse childhood experience (ACE) was measured by the ACE questionnaire developed in Kaiser-CDC study $[29,30]$. It includes 10 -item (yes/or) and the total score ranges from 0 to 10 with higher sum score indicating a higher likelihood of ACE. This instrument has been confirmed a good reliability and validity in Chinese population [31, 32], and Cronbach's alpha was 0.76 in this study.

Depression symptoms was assessed by The Centers for Epidemiological Studies Depression Scale (CESD) [33]. It is a 4-points Likert scale with 20 items and each item is scored from 0 (never) to 3 (always). A higher sum score indicates a severe level of depressions symptoms and this scale have shown good reliability and validity in Chinese population [34,35]. Cronbach's alpha was 0.87 in this study.

Identity concealment was assessed by a 6-item 5-points Likert subscale of nondisclosure [36], and each item is scored from 1 (never) to 5 (always) and total score ranges from 0 to 30 . Higher sum score indicates a higher likelihood of identity concealment. Cronbach's alpha was 0.91 in this study. 
Social support was measured by the multidimensional scale of social support [37]. It is a 7-points Likert scale including three dimensions (family support, friend support and others support) with 12 items. A higher total score indicates a higher level of social support [38]. Cronbach's alpha was 0.94 in this study.

Resilience was measured by Connor-Davidson Resilience scale-10 items (CD-RISC-10) [39]. Each item is rating from 0 (never) to 4 (always). The higher sum score indicates a higher level of resilience, with sum score ranging $0-40$. The scale has been reported a good reliability and validity $[40,41]$ and Cronbach's alpha was 0.95 in this study.

\section{HIV-related risky sexual behaviors}

We collected HIV-related risky sexual behaviors as follows: multiple sexual partner (2 or more male partners in the past six months were classified as multiple sexual partner), male partner type (all were fixed partners/all were one-night partners/both of them), sex after drinking (yes/no). HIV status of male partner were measured by the question "Do you have sex with man whose HIV status was positive in the past six month" and the options included yes, no or unsure. Inconsistent condom use was assessed by asking the frequency of condom use during having sex with men in the past six months, with options from 0 (never) to 4 (every time). 0-3 were classified as inconsistent condom use and 4 were classified as consistent condom use.

\section{Statistical analysis}

\section{Latent profile analysis (LPA)}

The latent profile analysis is a person-centered approach, aiming to find hidden group from measurable continuous variables. In addition, it is noteworthy that the pattern of complex identity attributes was used to characterize individuals and further divided into latent classes in LPA. In this study, psychosocial variables (sexual minority stigma, ACE, depression, identity concealment, social support, resilience) were incorporated into the LPA.

In order to explore the number of latent classes, a combination of model indexes need to be considered as follows: Log-likelihood, Bayesian information criterion (BIC), Adjust Bayesian information criterion (adj.BIC), Akaike information criterion (AIC). Lower Log-likelihood, BIC, adj.BIC and AIC indicated better model fit. Using the Lo-Mendel-Rubinto adjust bootstrap likelihood ratio test (LRT), if the statistical value less than 0.05 , it indicated this number model class $(\mathrm{K})$ were better than last one (K-1). Entropy index was used to determine the accuracy of latent profile model and higher entropy index showed higher accuracy of model. In order to increase the generalizability of LPA analysis, the minimum percentage of the classes were set 5\% [42]. LPA was conducted by Mplus 7.1 software.

\section{Logistic regression model}

After determining the potential classes of psychosocial factors, logistic regression model was used to explore the relationship between the latent pattern of psychosocial factors and HIV-related risky sexual behaviors. In details, multiple binary logistic regression models were performed to explore the 
associations between latent classes of psychosocial factors and multiple sexual partners/sex after drinking/inconsistent condom use. Multinominal logistic analysis were used to explore the associations between latent classes of psychosocial factors and male partner type/HIV status of male partner. In all logistic regression models, HIV-related risky behaviors were dependent variables, the latent pattern of psychosocial factors was independent variable, and socio-demographic variables were adjusted. Logistic regression model was conducted by SPSS 17.0 and statistical significance was set 0.05 (two-tails) in this study.

\section{Results}

\section{Characteristic of samples}

As shown in Table 1, the majority of participants (94.9\%) were Han ethnic with an average 27.14 $(S D=8.23$ ) years old. Of the all participants, $76.1 \%$ were college or higher, $84.4 \%$ were unmarried, and $89.5 \%$ were employed. Only $19.5 \%$ MSM reported their monthly income were more than 6000 Yuan (925\$). Most of MSM (72.3\%) were homosexual. 
Table 1

Basic socio-demographic characteristics of samples $(\mathrm{N}=589)$.

\begin{tabular}{|lll|}
\hline Characteristic & Number & Percent (\%) \\
\hline Age (Mean, SD) & $27.14 \pm 8.23$ \\
\hline Ethnicity & & \\
\hline Han group & 559 & 94.9 \\
\hline Others & 30 & 5.1 \\
\hline Education level & & \\
\hline High school or lower & 141 & 23.9 \\
\hline College or higher & 448 & 76.1 \\
\hline Marital status & & \\
\hline Unmarried & 497 & 84.4 \\
\hline Married/divorced & 92 & 15.6 \\
\hline Employment status & & \\
\hline Unemployed & 62 & 10.5 \\
\hline Employed & 527 & 89.5 \\
\hline Monthly income (RMB) & & \\
\hline$<1000$ Yuan & 70 & 11.9 \\
\hline 1000-3000 Yuan & 213 & 36.2 \\
\hline 3001-6000 Yuan & 191 & 32.4 \\
\hline$>6000$ Yuan & 115 & 19.5 \\
\hline Sexual orientation & & 72.3 \\
\hline Homosexual & 426 & 19.9 \\
\hline Bisexual & 117 & \\
\hline Unsure/heterosexual & 46 & \\
\hline
\end{tabular}

\section{Lpa Of Psychosocial Characteristics}

The number of latent class ranging from 1 class model to 4 class model was tested (see Table 2). LRT test confirmed that 3 class model was better than 2 class model, and 2 class model was better than 1 class model. However, 4 class model didn't show significantly better than 3 class model. And AIC index, 
BIC index and Log-likelihood index showed a lower amount of reduction after 3 class model. Therefore, 3 class model was the best class model in this study. In addition, we use Z-scores of psychosocial factors to compare with each other due to different measures. Figure 1 further showed the Z-scores of each psychosocial factor.

Table 2

Latent class fit indices.

\begin{tabular}{|lllllll|}
\hline & Log-likelihood & Entropy & AIC & BIC & Adj. BIC & Adj. LRT P-value \\
\hline 1 class model & - & - & 22663.92 & 22716.46 & 22678.36 & - \\
\hline 2 class model & -11168.64 & 0.68 & 22375.27 & 22458.46 & 22398.14 & 0.001 \\
\hline 3 class model & -11070.71 & 0.74 & 22193.41 & 22307.25 & 22224.71 & 0.015 \\
\hline 4 class model & -10999.46 & 0.78 & 22064.91 & 22209.40 & 22104.64 & 0.173 \\
\hline Note: AIC=Akaike information criteria BIC=Bayesian information criteria LRT=Lo-Mendell-Rubin test \\
\hline
\end{tabular}

Class 1, "social support and resilience group" (SR group), was consisted of 340 (57.7\%) individuals, social support was the highest in all psychosocial factors, resilience had the second highest score; ACE score, identity concealment, sexual minority stigma, and depression ranked the lowest in three model classes.

Class 2, "identity concealment group" (IC group), was consisted of 186 (31.6\%) individuals, and identity concealment was the highest point in all psychosocial factors, depression was the second highest score. In all three classes, sexual minority stigma and depression ranked 1, ACE and social support score ranked 2 , resilience ranked 3 .

Class 3, "ACE group", was consistent of $63(10.7 \%)$ individuals and ACE score was the highest point in all psychosocial factors, depression ranked 2 . In all three classes, identity concealment, sexual minority stigma, resilience and depression ranked 2, social support ranked 3.

\section{Logistics regression model of associations between psychosocial characteristic patterns and HIV-related risky behaviors}

As shown in Figure 2a, after controlling socio-demographic variables, logistic regression model revealed that IC group were more likely having one-night partner and fixed/one-night (all were one-night partners: $\mathrm{AOR}=2.74,95 \% \mathrm{Cl}=1.54,4.90$; fixed/one-night partner: $\mathrm{AOR}=2.01,95 \% \mathrm{Cl}=1.34-3.01$ ), having sex with male partner with unsure HIV status ( $A O R=2.12,95 \% \mathrm{Cl}=1.44-3.13$ ). Figure $2 \mathrm{~b}$ showed ACE group were more likely having inconsistent condom use $(\mathrm{AOR}=2.58,95 \% \mathrm{Cl}=1.41-4.73)$, and having sex with HIV-positive male partner $(\mathrm{AOR}=4.90,95 \% \mathrm{Cl}=1.95-12.30)$.

Moreover, in Figure 3, we further found that, compared with MSM having sex with HIV-negative male partner, those having sex with HIV-positive or HIV-unsure male partner were more likely to inconsistently 
use condom in ACE group $\left(\chi^{2}=6.21, P=0.045\right)$. However, it was not significant in other groups comparison.

\section{Discussion}

This study revealed six observable psychosocial characteristics were divided into three latent patterns (social support and resilience group, identity concealment group and ACE group) among HIV-negative MSM. "social support and resilience (SR) group" showed positive social support and resilience, while other two groups were contrary. "identity concealment (IC) group" had high points in identity concealment and depression, in addition, the sexual minority stigma score was not very low in this group. ACE group showed the highest ACE score, and the depression scores and sexual minority stigma score were also high. This aligned well with previous studies [27, 43, 44], showing that multiple psychosocial characteristics can coexist in one person and might influence each other.

Compared with "SR group", "IC group" were more likely having one-night male partner, and having sex with HIV-unsure male partner. Studies have shown that stigma can promote identity concealment and depression $[27,43,45]$, therefore, it is possible to explain why the IC group had higher scores of identity concealment and depression, and sexual minority stigma was not low. A current study has found that MSM concealing their identity had more occasional partners and less likely disclosed their HIV status to their partners, which was consistent with our findings [24]."IC group” had higher sexual orientation concealment scores, indicating that they were reluctant to disclose their information to their friends and acquaintances. They may seek one-night male partners so that they can avoid revealing their sexual orientation to friends and acquaintances. In addition, MSM with identity concealment are inclined to disclose their other information including HIV status [24], and also possibly ignore their casual partners' HIV status. Unaware of sex partner's HIV status was an important barrier to reduce the transmission of HIV infection [15, 46]. It has been shown that HIV status of male sex partner play an important role in HIV risk assessment, especially having sex with HIV-unsure male partner might have greater risk [47]. In HIV intervention targeted at MSM in the "IC group", psychological counseling need be emphasized, and HIV risk awareness education is critical for promoting MSM to proactively understand HIV status of sexual partners and consistently use condoms.

"ACE group" were more likely having inconsistent condom use and HIV-positive male partner. Previous studies [48-50] have shown that MSM with higher level of ACE exposure were more likely to feel psychological distress (e.g., depression), and in turn pay less attention to HIV infection, increasing the risk of infecting HIV. And it has been revealed that ACE exposure was associated with condomless anal intercourse [20], which was consistent with our findings. It was also found that "ACE group" were more likely to have HIV-positive male partners, and further analysis showed $90 \%$ of MSM in this subgroup reporting ever having sex with HIV-positive partner inconsistently used condoms. This suggested that for MSM in this subgroup, HIV intervention need focus on consistent condom use attitude and behavior. 
Though we used a unique approach to explore HIV-related risky sexual behavior among MSM, there were some limitations in this study. First, this was a cross-sectional study and causal relationships between psychosocial factors and HIV risk behaviors cannot be determined. Second, the study subjects were obtained through non-random sampling, so there may be selection bias. Finally, we only chose several psychosocial characteristics basing on previous studies, other psychosocial variables (e.g., anxiety) were not included in the latent class model, and maybe change the latent profile.

\section{Conclusion}

Basing six important psychosocial factors of depression, resilience, social support, adverse childhood experience, sexual minority stigma, and identity concealment, MSM were divided into three latent pattern classes. Compared with "social support and reliance group", "identity concealment group" and "ACE group" were more likely to engage in HIV-related risky sexual behaviors (e.g., inconsistent condom use). In HIV intervention targeted on MSM, psychosocial characteristics need to be focused on, especially paying more attention to MSM with higher level of identity concealment or ACE. Besides mental health guidance, promoting serological disclosure of sexual partners and consistent condom use behavior is critical for MSM with higher level of identity concealment or ACE.

\section{Declarations}

\section{Ethical Approval and Consent to participate}

All participants provided written consent and this study was approved by the Medical Ethics Committee at Wuhan University, China.

\section{Consent for publication}

Not applicable.

\section{Availability of data and materials}

The datasets analysed during the current study are not publicly available due [Further follow-up investigations are ongoing] but are available from the corresponding author on reasonable request.

\section{Competing interests}

There is no conflict of interest for all authors.

\section{Funding}

This study was supported by the National Natural Science Foundation of China (Grant No. 81673196). The funder had no role in study design, data collection, analysis, and interpretation of this manuscript.

\section{Authors' contributions}


MZ, ZD analyzed this data and wrote manuscript; JT, BZ, QJ, BY collected this data; HY and ZD revised this manuscript; $\mathrm{HY}$ designed this research and revised manuscript; $\mathrm{XC}$ edited the language. All authors approved the publication of this manuscript.

\section{Acknowledgements}

We would like to thank all participants who generously shared their time and the organizations for their help in data collection.

\section{Authors' information}

Address correspondence to Dr. Hong Yan, School of Health Sciences, Wuhan University, Wuhan, Hubei Province, China; Email: yanhmjxr@whu.edu.cn

\section{References}

1. Zeng X, Zhong X, Peng B, Zhang Y, Kong C, Liu X, et al. Prevalence and associated risk characteristics of HIV infection based on anal sexual role among men who have sex with men: a multi-city crosssectional study in Western China. International Journal of Infectious Diseases. 2016;49:111-8. doi:https://doi.org/10.1016/j.ijid.2016.06.009.

2. Beyrer C, Baral SD, van Griensven F, Goodreau SM, Chariyalertsak S, Wirtz AL, et al. Global epidemiology of HIV infection in men who have sex with men. Lancet. 2012;380(9839):367-77. doi:10.1016/s0140-6736(12)60821-6.

3. Chow EP, Tucker JD, Wong FY, NehI EJ, Wang Y, Zhuang X, et al. Disparities and risks of sexually transmissible infections among men who have sex with men in China: a meta-analysis and data synthesis. PLoS One. 2014;9(2):e89959. doi:10.1371/journal.pone.0089959.

4. Prevalence and awareness of HIV infection among men who have sex with men - 21 cities, United States, 2008. MMWR Morb Mortal Wkly Rep. 2010;59(37):1201-7.

5. Report CARP. National Health and Family Planning Commission of the People's Republic of China. 2015.

6. Zhang L, Ding X, Lu R, Feng L, Li X, Xiao Y, et al. Predictors of HIV and syphilis among men who have sex with men in a Chinese metropolitan city: comparison of risks among students and non-students. PLoS One. 2012;7(5):e37211. doi:10.1371/journal.pone.0037211.

7. de Voux A, Bernstein KT, Kirkcaldy RD, Zlotorzynska M, Sanchez T. Self-Reported Extragenital Chlamydia and Gonorrhea Testing in the Past 12 Months Among Men Who Have Sex with Men in the United States-American Men's Internet Survey, 2017. Sex Transm Dis. 2019;46(9):563-70. doi:10.1097/olq.0000000000001032.

8. Wang Y, Wang Z, Jia M, Liang A, Yuan D, Sun Z, et al. Association between a syndemic of psychosocial problems and unprotected anal intercourse among men who have sex with men in Shanghai, China. BMC Infect Dis. 2017;17(1):46. doi:10.1186/s12879-016-2132-8. 
9. Yuan R, Cheng H, Chen LS, Zhang X, Wang B. Prevalence of different HIV-1 subtypes in sexual transmission in China: a systematic review and meta-analysis. Epidemiol Infect. 2016;144(10):2144-53. doi:10.1017/s0950268816000212.

10. Huynh K, Gulick PG. HIV Prevention. StatPearls. Treasure Island (FL): StatPearls Publishing. Copyright (C) 2021, StatPearls Publishing LLC.; 2021.

11. Ma Q, Zeng S, Xia S, Pan X, Wang D, Zhu H, et al. Risky sexual networks and concentrated HIV epidemics among men who have sex with men in Wenzhou, China: a respondent-driven sampling study. BMC Public Health. 2015;15:1246. doi:10.1186/s12889-015-2591-7.

12. Zhao J, Chen L, Cai WD, Tan JG, Tan W, Zheng CL, et al. HIV infection and sexual behaviors among non-commercial men who have sex with men at different venues. Arch Sex Behav. 2014;43(4):8019. doi:10.1007/s10508-013-0167-0.

13. Serovich JM, Laschober TC, Brown MJ, Kimberly JA. Longitudinal Findings on Changes in and the Link Between HIV-Related Communication, Risky Sexual Behavior, and Relationship Status in Men Who Have Sex With Men Living With HIV. Sex Transm Dis. 2017;44(12):732-8. doi:10.1097/olq.0000000000000680.

14. Guo W, Wu ZY, Song AJ, Poundstone K. Impact of HIV/sexually transmitted infection testing on risky sexual behaviors among men who have sex with men in Langfang, China. Chin Med J (Engl). 2013;126(7):1257-63.

15. lakunchykova O, Burlaka V, King EJ. Correlates of Serosorting and Knowledge of Sexual Partner's HIV Status Among Men Who have Sex with Men in Ukraine. AIDS Behav. 2018;22(6):1955-64. doi:10.1007/s10461-017-2002-4.

16. Thompson RG Jr, Eaton NR, Hu MC, Grant BF, Hasin DS. Regularly drinking alcohol before sex in the United States: effects of relationship status and alcohol use disorders. Drug Alcohol Depend. 2014;141:167-70. doi:10.1016/j.drugalcdep.2014.05.021.

17. Li D, Li C, Wang Z, Lau JT. Prevalence and associated factors of unprotected anal intercourse with regular male sex partners among HIV negative men who have sex with men in China: a crosssectional survey. PLoS One. 2015;10(3):e0119977. doi:10.1371/journal.pone.0119977.

18. Wong CF, Schrager SM, Holloway IW, Meyer IH, Kipke MD. Minority stress experiences and psychological well-being: the impact of support from and connection to social networks within the Los Angeles House and Ball communities. Prev Sci. 2014;15(1):44- 55. doi:10.1007/s11121-0120348-4.

19. Ahaneku H, Ross MW, Nyoni JE, Selwyn B, Troisi C, Mbwambo J, et al. Depression and HIV risk among men who have sex with men in Tanzania. AIDS care. 2016;28(Suppl 1(sup1):140-7. doi:10.1080/09540121.2016.1146207.

20. Bertolino DF, Sanchez TH, Zlotorzynska M, Sullivan PS. Adverse childhood experiences and sexual health outcomes and risk behaviors among a nationwide sample of men who have sex with men. Child Abuse Negl. 2020;107:104627. doi:10.1016/j.chiabu.2020.104627. 
21. Burnham KE, Cruess DG, Kalichman MO, Grebler T, Cherry C, Kalichman SC. Trauma symptoms, internalized stigma, social support, and sexual risk behavior among HIV-positive gay and bisexual MSM who have sought sex partners online. AIDS care. 2016;28(3):347-53. doi:10.1080/09540121.2015.1096894.

22. McNair OS, Gipson JA, Denson D, Thompson DV, Sutton MY, Hickson DA. The Associations of Resilience and HIV Risk Behaviors Among Black Gay, Bisexual, Other Men Who Have Sex with Men (MSM) in the Deep South: The MARI Study. AIDS Behav. 2018;22(5):1679-87. doi:10.1007/s10461017-1881-8.

23. Logie CH, Wang Y, Marcus NL, Levermore K, Jones N, Ellis T, et al Pathways From Sexual Stigma to Inconsistent Condom Use and Condom Breakage and Slippage Among MSM in Jamaica. Journal of acquired immune deficiency syndromes (1999). 2018;78(5):513-21. doi:

10.1097/qai.0000000000001712.

24. Pachankis JE, Hatzenbuehler ML, Hickson F, Weatherburn P, Berg RC, Marcus U, et al Hidden from health: structural stigma, sexual orientation concealment, and HIV across 38 countries in the European MSM Internet Survey. AIDS (London, England). 2015;29(10):1239-46. doi: 10.1097/qad.0000000000000724.

25. Li X, Guo M, Wang W, Wei L, Xiao C, Yu L, et al. Association between childhood sexual abuse and patterns of HIV risky sexual behaviors among men who have sex with men in China: A latent class analysis. Child Abuse Negl. 2021;120:105164. doi:10.1016/j.chiabu.2021.105164.

26. Yan H, Li X, Li J, Wang W, Yang Y, Yao X, et al. Association between perceived HIV stigma, social support, resilience, self-esteem, and depressive symptoms among HIV-positive men who have sex with men (MSM) in Nanjing, China. AIDS care. 2019;31(9):1069-76. doi:10.1080/09540121.2019.1601677.

27. Ding C, Chen X, Wang W, Yu B, Yang H, Li X, et al. Sexual Minority Stigma, Sexual Orientation Concealment, Social Support and Depressive Symptoms Among Men Who have Sex with Men in China: A Moderated Mediation Modeling Analysis. AIDS Behav. 2020;24(1):8-17. doi:10.1007/s10461-019-02713-3.

28. Neilands TB, Steward WT, Choi KH. Assessment of stigma towards homosexuality in China: a study of men who have sex with men. Arch Sex Behav. 2008;37(5):838-44. doi:10.1007/s10508-007-9305$X$.

29. Kaiser-CDC. Centers for Disease Control and Prevention: adverse childhood experiences. (ACE) study. Available from: http://www.cdc.gov/ace/about.htm. Accessed April 32020.

30. Felitti VJ, Anda RF, Nordenberg D, Williamson DF, Spitz AM, Edwards V, et al. Relationship of childhood abuse and household dysfunction to many of the leading causes of death in adults. The Adverse Childhood Experiences (ACE) Study. Am J Prev Med. 1998;14(4):245-58. doi:10.1016/s0749-3797(98)00017-8.

31. Liu Z, Yang Y, Shi Z, Liu J, Wang Y. The risk of male adult alcohol dependence: The role of the adverse childhood experiences and ecological executive function. Compr Psychiatr. 2016;68:129-33. 
doi:10.1016/j.comppsych.2016.04.006.

32. Fung HW, Ross CA, Yu CK, Lau EK. Adverse childhood experiences and dissociation among Hong Kong mental health service users. Journal of trauma dissociation: the official journal of the International Society for the Study of Dissociation (ISSD). 2019;20(4):457-70. doi:10.1080/15299732.2019.1597808.

33. Radloff LS. The CES-D, Scale:A Self-Report Depression Scale for Research in the General Population. 1977;1(3):385-401. doi: 10.1177/014662167700100306.

34. Wang W, Xiao C, Yao X, Yang Y, Yan H, Li S. Psychosocial health and suicidal ideation among people living with HIV/AIDS: A cross-sectional study in Nanjing, China. PLoS One. 2018;13(2):e0192940. doi:10.1371/journal.pone.0192940.

35. Jie WZFG. Development of the Chinese age norms of CES-D in urban area. Chin Ment Health J (Chinese). 2010;2:139-43.

36. Testa R, Habarth J, Peta J, Balsam K, Bockting W. Development of the Gender Minority Stress and Resilience Measure. Psychology of Sexual Orientation Gender Diversity. 2015;2:65-77. doi:10.1037/sgd0000081.

37. Zimet GD, Powell SS, Farley GK, Werkman S, Berkoff KA. Psychometric characteristics of the Multidimensional Scale of Perceived Social Support. J Pers Assess. 1990;55(3-4):610-7. doi:10.1080/00223891.1990.9674095.

38. Li S, Li L, Zheng H, Wang Y, Zhu X, Yang Y, et al. Relationship between multifaceted body image and negative affect among women undergoing mastectomy for breast cancer: a longitudinal study. Arch Womens Ment Health. 2018;21(6):681-8. doi:10.1007/s00737-018-0860-z.

39. Ehrich J, Mornane A, Powern T. Psychometric Validation of the 10-item Connor-Davidson Resilience Scale. J Appl Meas. 2017;18(2):122-36.

40. Ding C, Wang T, Chen X, Li J, Wang W, Huang D, et al. Association of Adverse Childhood Experience and Attention Deficit Hyperactivity Disorder with depressive symptoms among men who have sex with men in China: moderated mediation effect of resilience. BMC Public Health. 2019;19(1):1706. doi:10.1186/s12889-019-8016-2.

41. Zhang M, Zhang J, Zhang F, Zhang L, Feng D. Prevalence of psychological distress and the effects of resilience and perceived social support among Chinese college students: Does gender make a difference? Psychiatry Res. 2018;267:409-13. doi:10.1016/j.psychres.2018.06.038.

42. Nylund KL, Asparouhov T, Muthén B. Deciding on the Number of Classes in Latent Class Analysis and Growth Mixture Modeling: A Monte Carlo Simulation Study. Structural Equation Modeling A Multidisciplinary Journal. 2007;14(4):535-69.

43. Stahlman S, Grosso A, Ketende S, Sweitzer S, Mothopeng T, Taruberekera N, et al. Depression and Social Stigma Among MSM in Lesotho: Implications for HIV and Sexually Transmitted Infection Prevention. AIDS Behav. 2015;19(8):1460-9. doi:10.1007/s10461-015-1094-y.

44. Bockting WO, Miner MH, Swinburne Romine RE, Hamilton A, Coleman E. Stigma, mental health, and resilience in an online sample of the US transgender population. American journal of public health. 
2013;103(5):943-51. doi:10.2105/ajph.2013.301241.

45. Kutner BA, Simoni JM, Aunon FM, Creegan E, Balán IC. How Stigma Toward Anal Sexuality Promotes Concealment and Impedes Health-Seeking Behavior in the U.S. Among Cisgender Men Who Have Sex with Men. Arch Sex Behav. 2021;50(4):1651-63. doi:10.1007/s10508-019-01595-9.

46. Fujimoto K, Williams ML, Ross MW. A network analysis of relationship dynamics in sexual dyads as correlates of HIV risk misperceptions among high-risk MSM. Sex Transm Infect. 2015;91(2):130-4. doi:10.1136/sextrans-2014-051742.

47. Golden MR, Brewer DD, Kurth A, Holmes KK, Handsfield HH. Importance of sex partner HIV status in HIV risk assessment among men who have sex with men. Journal of acquired immune deficiency syndromes (1999). 2004;36(2):734-42. doi: 10.1097/00126334-200406010-00011.

48. Giano Z, Hubach RD. Adverse childhood experiences and mental health: Comparing the link in rural and urban men who have sex with men. J Affect Disord. 2019;259:362-9. doi:10.1016/j.jad.2019.08.044.

49. Suarez NA, Peitzmeier SM, Potter J, Samandur A, Reisner SL. Preliminary findings for adverse childhood experiences and associations with negative physical and mental health and victimization in transmasculine adults. Child Abuse Negl. 2021;118:105161. doi:10.1016/j.chiabu.2021.105161.

50. Ports KA, Lee RD, Raiford J, Spikes P, Manago C, Wheeler DP. Adverse Childhood Experiences and Health and Wellness Outcomes among Black Men Who Have Sex with Men. Journal of urban health: bulletin of the New York Academy of Medicine. 2017;94(3):375-83. doi:10.1007/s11524-017-0146-1.

\section{Figures}

SR group ACE group


Figure 1

Latent classes by categorical Z-score mean.
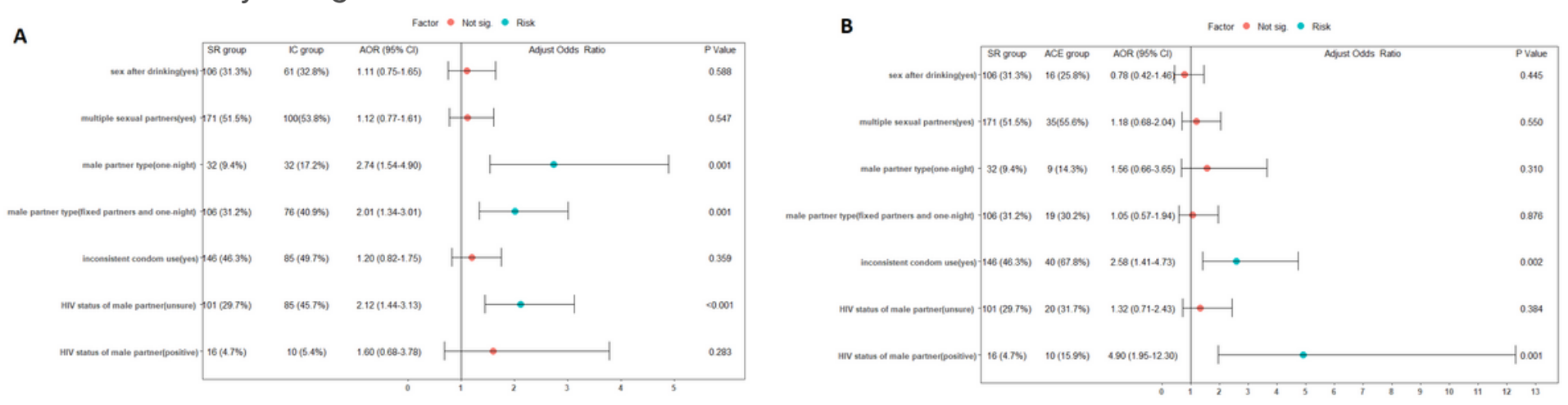

\section{Figure 2}

a, The association between IC psychosocial characteristics and HIV-related risky sexual behaviors (Multivariate logistic regression analysis, SR psychosocial characteristics as ref). Note: adjust for all basic socio-demographic variables. $2 \mathrm{~b}$, The association between ACE psychosocial characteristics and HIV-related risky sexual behaviors (Multivariate logistic regression analysis, SR psychosocial characteristics as ref). Note: adjust for all basic socio-demographic variables.

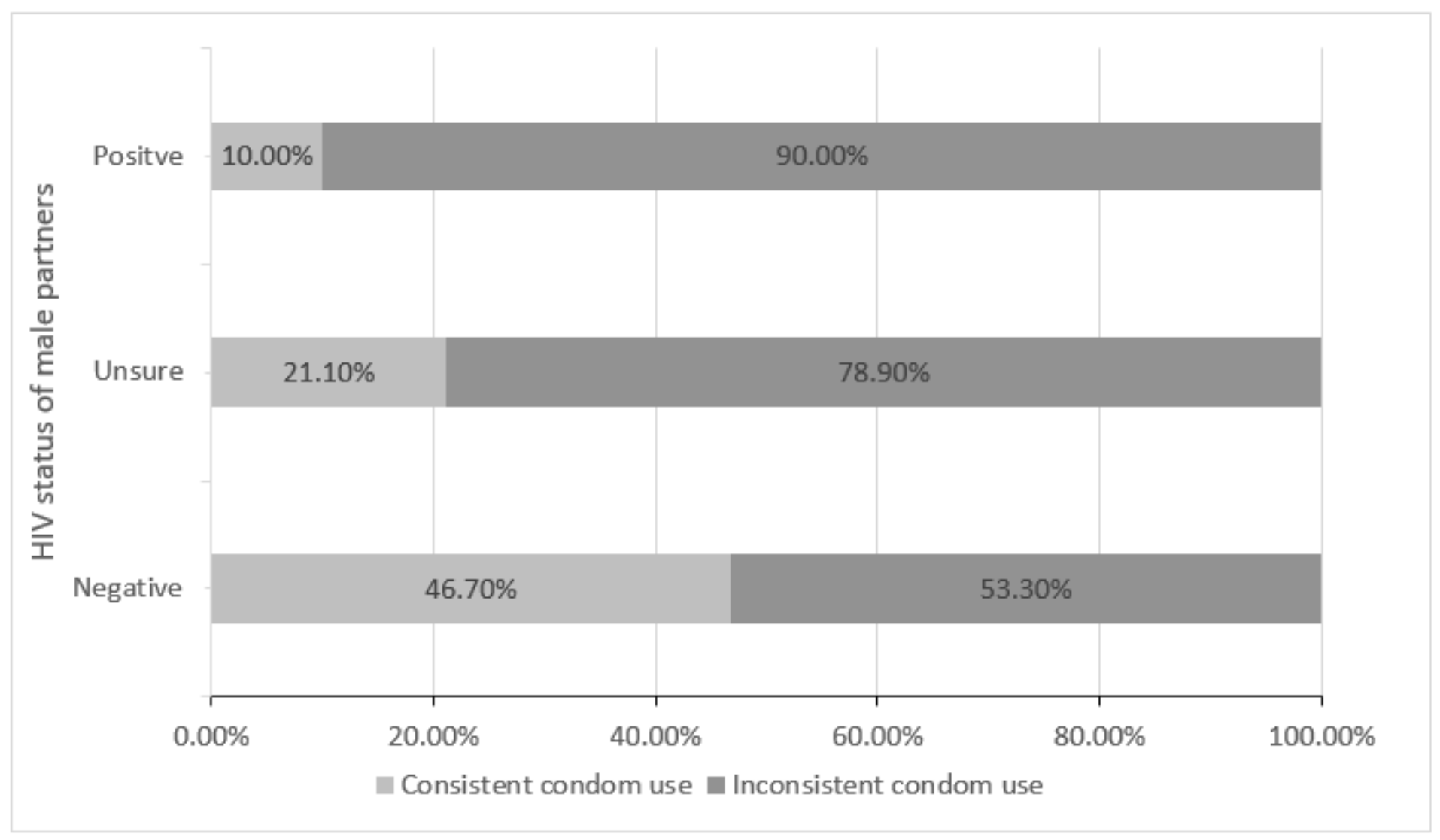

\section{Figure 3}


Inconsistent condom use rate of different HIV status of male partner among the individual who was classified as ACE group $(\chi 2=6.21, P=0.045)$ 